\title{
Properties of Antiprotons and Antihydrogen, and the Study of Exotic Atoms
}

\author{
Michael Doser \\ CERN, EP-1211 Geneva, Switzerland \\ michael.doser@cern.ch
}

\begin{abstract}
The study of exotic atoms, of antiprotons and of antihydrogen atoms provides many windows into the investigation of fundamental symmetries, of interactions between particles and nuclei, of nuclear physics and of atomic physics. This field appeared at CERN simultaneously with the first accelerators, and has advanced over the decades in parallel with improvements and advances in its infrastructure.
\end{abstract}

\section{Introduction}

Since the earliest days of CERN, antiprotons and exotic atoms have formed a central part of its experimental program. Complementary to particle physics approaches, studies of exotic atoms have played an important role in probing particle properties (particle mass determinations), in studying the strong interaction at relatively large distances of several fm (in pionic or antiprotonic atoms, for example), in investigating nuclear physics (through measurements of atomic transitions of pionic or kaonic atoms comprised of different nuclei) or nuclear radii (with muonic atoms), or in tests of fundamental symmetries (measurements of mass, charge and magnetic moments of antiprotons in antiprotonic helium).

At the same time, antiprotons which were used initially as a workhorse in the study of mesonic states, before techniques to accumulate and store them were developed in the 1970s and 1980s, have since then been studied at very high precision, both as individual particles in traps, as well as in the form of atoms consisting completely of antimatter. Here too, the goals are similar: search for violation of symmetries like CPT through precision spectroscopy of antihydrogen atoms or precision comparisons of mass, charge and magnetic moment between antiprotons and protons, and tests of the weak equivalence principle through measurements of the gravitational interaction of (neutral) antihydrogen atoms.

The study of these exotic systems does not quite follow the general history of CERN: while accelerators at CERN became ever more powerful over the decades, the energies required to produce the sufficiently long-lived particles (muons, pions, kaons and antiprotons) that form the building blocks of exotic atoms are modest; even today, the same Proton Synchrotron (PS, Fig. 1) that produced the first antiprotons at CERN in $1960,{ }^{1}$ shortly after its start-up in 1959 still 


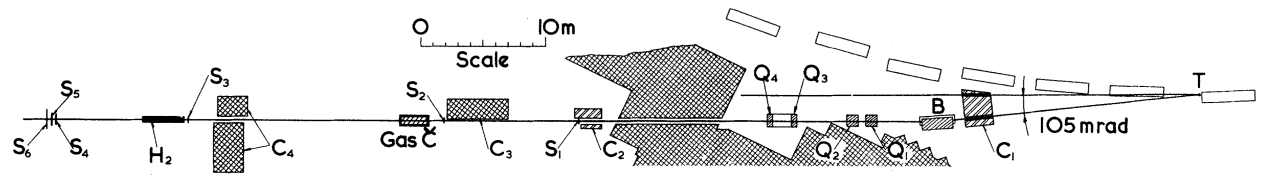

\section{$\$$ Concrete}

\section{Lead}

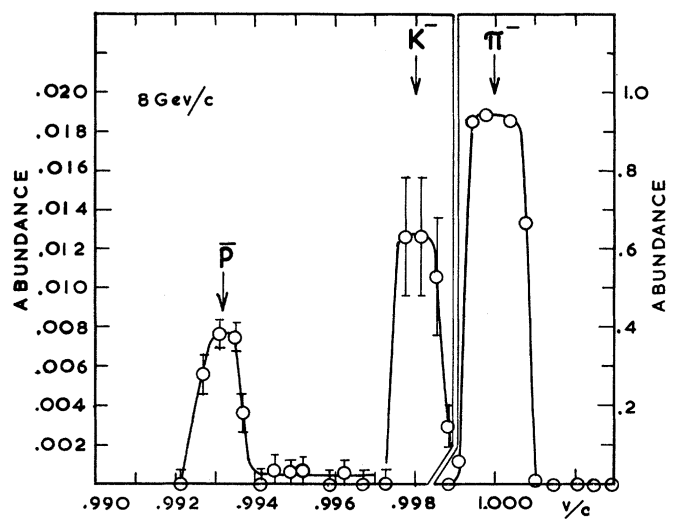

Fig. 1. Top: First antiproton beam line at CERN (1960). The dashed line to the right of the shielding blocks is the Proton Synchrotron. ${ }^{1}$ Bottom: beam composition in this beam line. ${ }^{1}$

continues to provide the antiprotons that form the heart of experiments on them or on antihydrogen atoms, and muonic and pionic atoms are now studied at highintensity (but low energy) accelerators outside of CERN. Nevertheless, technical developments at CERN have played a defining role in the study of exotic atoms and of antiprotons. In particular, experiments relying on antiprotons have only become possible through the invention of stochastic cooling, the construction of a dedicated storing and cooling accelerator infrastructure (the Antiproton Accumulator (AA) and the Antiproton Collector (AC)), the development of antiproton trapping techniques in the 1980s at CERN's dedicated antiproton experimental facility LEAR (low energy antiproton ring) from 1982 to 1996, and - since 2000 - of the transformation of the AC into the unique Antiproton Decelerator (AD) facility, which hosts all existing experiments worldwide that require trapped antiprotons.

\section{Pionic, Muonic, Kaonic and other Exotic Atoms}

Negatively charged particles with a sufficiently long lifetime (relative to the atomic processes involved with capture and decay of the resultant atoms to deeply bound states) offer a window to study atomic physics processes (Rydberg states, cascades, binding energies, lifetimes), but also nuclear physics processes: the deeply bound states' energy levels and lifetimes are affected by strong-interaction effects, which 
in turn provide the opportunity to study nuclear forces at large distances ("nuclear stratosphere") as well as isotope-related nuclear deformations. As the capture and subsequent de-excitation process occurs on time-scales of $\mathrm{ps} \sim \mathrm{ns},{ }^{2}, 3$ muons, pions, kaons, antiprotons, but also shorter-lived baryons, such as $\Sigma^{-}$, or even potentially $\Xi^{-}$and $\Omega^{-}$, can form exotic relatively long-lived atoms (although $\Xi^{-}$or $\Omega^{-}$exotic atoms have to date not been observed).

The first such measurements at CERN started at the SC in 1961: observation of $2 \mathrm{p}-1 \mathrm{~s}$ transitions in muonic atoms ${ }^{4}$ and subsequently in pionic atoms. ${ }^{5}$

With the advent of intense $\mathrm{K}^{-}$beams in the late $1960 \mathrm{~s}$, counter experiments on hypernuclei became possible. While the focus was mainly on hypernuclear continuum states, the study of kaonic atoms also took place at CERN: shift and width of the $1 \mathrm{~s}$ level of the $\mathrm{K}^{-} \mathrm{p}$ atom occupied a number of experiments at the PS, ${ }^{6}$ leading also to the first observations, and then studies, of $\Sigma^{-}$exotic atoms from 1970 onward. ${ }^{7}$ In the same year, also the first X-ray transitions of antiprotonic atoms were detected and investigated by the same group. ${ }^{8}$

Although earlier observations of exotic atoms had been made, the contemporaneous development of solid-state detectors - by allowing high resolution $\gamma$-ray detection — made precision spectroscopy possible, and with it precise determinations of energies (and thus of minute energy shifts), of natural line-widths and of intensities for a large range of transitions and nuclei possible.

\subsection{Atomic physics}

Through their unusual composition, but also through the formation process, the physics involved in de-excitation, and the much smaller radii of the ground state levels (and thus a strongly enhanced proximity to the nucleus), exotic atoms are an ideal test bed for aspects of atomic physics that are significantly more difficult to probe in conventional atoms.

Leaving aside the more complex highly excited exotic atoms, where the heavy negative particle shell radius is larger than the lowest electron Bohr radius, the exotic atom can be considered hydrogen-like, with bound state energy eigenvalues of

$$
E(n, l)=\frac{m_{x}}{m_{e}} E_{0}(n, l)
$$

with $m_{x}$ the reduced mass of the exotic atom with the negative particle, $m_{e}$ the electron mass, and $E_{0}$ the energy levels of the ordinary atom. The resulting Bohr radius is consequently (since the mass ratio for even the lightest such negative particle - the muon - is 200) comparable to or even smaller than the radius of the nucleus. For an exotic atom with nuclear charge $Z$ and containing a negative particle of mass $m_{x}$, the fine structure splitting is given by:

$$
\Delta E=\left(\mu_{D}+2 \mu_{a}\right) \frac{(Z \alpha)^{4}}{2 n^{3}} \frac{m_{x} c^{2}}{l(l+1)}
$$


with $\mu_{D}$ the Dirac moment and $\mu_{a}$ the anomalous magnetic moment. ${ }^{9}$ Measurement of the transition energies (proportional to the mass of the negative particle) and of the fine structure splitting (proportional to its magnetic moment) thus provide access to the negative particles properties. Depending on whether the negative particle is a spin- $\frac{1}{2}$ particle $\left(\mu^{-}, \overline{\mathrm{p}}, \Sigma^{-}\right)$or a meson $\left(\pi^{-}, \mathrm{K}^{-}\right)$, different corrections need to be applied. Hadronic negative particles will be sensitive to the distribution of hadronically interacting particles in the nucleus; leptonic probes will be sensitive to the (related) charge distribution in the nucleus, once their Bohr radius is sufficiently close to the nuclear surface to interact with it.

\subsubsection{Formation processes}

The formation of exotic atoms, described first by Fermi and Teller, ${ }^{10}$ and subsequently detailed by Ponomarev, ${ }^{11}$ consists of a series of steps: the negative meson (or baryon) slows down from relativistic velocities to velocities of the order of atomic electron velocities; at this point, the negative particle is captured into a highly excited (Rydberg) state, with principal quantum number dependent on the particle mass and ejects an electron. The capture cross-section is related to the overlap between the wave functions of the particle and the atomic electron ${ }^{11}$ so the heavy particle will initially populate atomic states with radii close to that of the (ejected) electrons. The observation of metastable antiprotonic atoms allows probing this process: the PS205 experiment at LEAR (see Section 4.1) was able to follow the trapping of antiprotons into the large- $n$ and large- $l$ metastable states of neutral antiprotonic helium. The principal quantum number $n_{0}$ of these initial states is expected to be

$$
n_{0} \sim\left(m_{x} / m_{e}\right)^{1 / 2} \sim 40
$$

where $m_{x}$ is the reduced mass of the exotic atom with the negative particle, and $m_{e}$ the electron mass. The first observed laser-induced transition in antiprotonic helium $^{12}$ corresponds to a transition $(n, l)=(39,35) \rightarrow(38,34)$, in agreement with the expected population of high- $n$ states.

\subsubsection{De-excitation processes}

The formed exotic atoms have several possible de-excitation pathways: radiative transitions, Auger effect, or Stark transitions $(n, l) \rightarrow(n, l-1)$ through collisions with other atoms. The details of the de-excitation depend on many parameters (target density, type of negative particle, etc.) and the final states populated depend sensitively on these (for kaonic atoms, for example, see Ref. 13). Measurements of cascade times for $\mathrm{K}^{-},{ }^{14}$ for $\Sigma^{-15}$ or for antiprotons, ${ }^{16,17}$ as well as searches for X-ray transitions into the lowest-lying s- and p-states in pionic, kaonic, $\Sigma^{-}$ and antiprotonic atoms, were carried out in a number of experiments at CERN, and confirm the details of the cascade calculations, in particular with regards to the importance of Stark transitions, which dramatically shorten the cascade time, 
and populate high- $n$, low- $l$ states in the course of the cascade. A consequence of this is that in bubble chamber experiments, antiproton annihilation occurs almost exclusively from the s-wave, with implications on the distribution of final states produced in these annihilations.

\subsection{Particle parameters}

Masses, charges and magnetic moments of the negatively-charged particles in exotic atoms determine the transition energies. Compared to measurements based on kinematics $\left(\pi \rightarrow \mu \nu\right.$, or $\left.\mathrm{K}^{-} \rightarrow \pi^{+} \pi^{-} \pi^{-}\right)$, spectroscopic measurements can reach higher precision (although calibration issues are of concern).

The first precision measurement of the pion mass via $4 \mathrm{f}-3 \mathrm{~d}$ X-ray lines in Ca and Ti exotic atoms using a crystal spectrometer ${ }^{18}$ took place at Berkeley in 1967, and the same technique was also employed there in kaonic atoms (4f-3d X-ray lines in $\mathrm{Cl}$ ) to provide the first spectroscopic determination of the negative kaon mass. ${ }^{19}$ Subsequent exotic-atom determinations of mass or magnetic moments of $\mathrm{K}^{-},{ }^{20} \overline{\mathrm{p}}^{9}$ and $\Sigma^{-21}$ were carried out at Brookhaven's AGS from 1975. However, the advent of improved detection techniques (a higher efficiency $\mathrm{Ge}(\mathrm{Li})$ detector), improvements in the accuracy and reliability of calculations of corrections to the energy levels of mesonic atoms (allowing also to choose less sensitive transitions), and better calibration lines allowed an improved measurement of the pion mass to be carried out at CERN's SC in 1971 by Backenstoss et al. ${ }^{22}$ allowing them also to provide the first upper limit to the mass of the $\nu_{\mu}$ of less than $1 \mathrm{MeV}$. The start-up of the PS at CERN was also immediately used by the same group to carry out a 4 -fold improved determination of the $\mathrm{K}^{-}$mass via kaonic $\mathrm{X}$-rays in $\mathrm{Au}$ and $\mathrm{Ba}^{23}$

The special case of measurements of the antiproton's mass, charge and magnetic moment in antiprotonic helium (which affords far higher precision than is possible when measuring energies of X-rays emitted during a cascade) is dealt with in Sections 4.1 and 4.2 .

\subsection{The strong interaction}

Depending on the type of the negatively-charged particle in the exotic atoms, strong interaction effects may affect the lowest-lying bound states, shifting their energy level, modifying the lifetime of the state, and changing the transition probabilities (and thus the transition intensities) with respect to a pure QED reference value. A number of probes are also sensitive to the presence of a diffuse neutron halo at the nuclear surface.

A detailed understanding of the strong interaction became possible in part through improved understanding of the $\mathrm{K}^{-} \mathrm{N}$ and $\pi^{-} \mathrm{N}$ interactions as probed in the deeply-bound pionic and kaonic atoms, as the strong interaction will induce an energy shift and broadening of these states. Pionic $2 \mathrm{p}-1 \mathrm{~s}$ transitions in $\mathrm{Be},{ }^{10} \mathrm{~B}$, $\mathrm{C}, \mathrm{N},{ }^{16} \mathrm{O},{ }^{18} \mathrm{O}, \mathrm{F}$ and $\mathrm{Na}^{5}$ at CERN's $\mathrm{SC}$ were among the first to have sufficient 
sensitivity to detect the 1 s level shifts and widths and to establish the presence of isospin dependence (Fig. 2).

The same group also carried out the first measurements in a kaonic X-ray transition of the natural linewidth and energy shift due to the strong kaon-nuclear interaction, ${ }^{6,27}$ which were instrumental in ruling out a number of mechanisms proposed to explain an increase of the $\mathrm{K}^{-}$absorption in nuclear matter, among them the need for an attractive real part in the K-nucleus optical potential (rather than the repulsive real part suggested by $\mathrm{K}-\mathrm{N}$ scattering), or an extended neutron halo above the nucleus. A number of subsequent investigations have clarified this point: the presence of a nuclear resonance (the $\Lambda(1405)$ s-wave resonance in the isospin $I=0$ channel) strongly affects this amplitude; the sub-threshold $\mathrm{K}-\mathrm{p}$ interaction dominates over the $\mathrm{K}-\mathrm{n}$ interaction.

A rather recent (late 1990s) experiment at CERN to probe QCD at very low energies studied fully-exotic hydrogen-like atoms in which both participants are either pions or kaons. The DIRAC experiment at the PS focused first on observing such atoms (produced through electromagnetically-interacting, kinematicallymatched opposite-charge pions produced in $24 \mathrm{GeV}$ p-N interactions), and then determining the lifetime of $\pi^{+} \pi^{-}$atoms. The corresponding measurement ${ }^{29}$ of this lifetime of $\tau_{2 \pi}=\left(3.15_{-0.19}^{+0.20}(\text { stat })_{-0.18}^{+0.20}(\mathrm{sys})\right)$ fs represents a very sensitive test of Chiral Perturbation Theory (ChPT). Similar $\pi \mathrm{K}$ atoms probe the more general 3-flavour SU(3) structure of low-energy hadronic interactions that is not accessible to $\pi \pi$ atoms. The first observation of these atoms ${ }^{30}$ only allows setting a lower limit of $0.8 \mathrm{fs}$ (at $90 \% \mathrm{CL}$ ) for these atoms whose lifetime is predicted by ChPT to be $3.7 \pm 0.4$ fs. $^{31}$

\subsection{Nuclear physics}

Shape and charge distributions of the nucleus can be probed through an accurate measurement of the energy and, to a lesser degree, the relative intensities of X-rays in muonic atoms. In a series of measurements beginning in 1961, ${ }^{4}$ Backenstoss and collaborators - using the $\mu$-channel of the SC, which provided an intense and rather pure beam of muons - carried out a systematic exploration of nuclei. Electrical charge distributions of nuclei are best measured through muonic X-ray transitions and electron scattering experiments. The lowest levels of the muonic atom provide the highest sensitivity to the charge distribution, and this sensitivity increases with increasing $Z$. Often using the $2 \mathrm{p}-1$ s transition as their workhorse to determine the energy shifts and intensities of the transitions, this group deduced nuclear charge distribution parameters for, e.g. spherical nuclei from $\mathrm{Cl}$ to $\mathrm{Pb} .{ }^{32,} 33$ This method is of course also applicable to deformed nuclei, such as Sm, Eu, Tb, Ho, Hf, Ta, W, Os, Ir, Th, ${ }^{233} \mathrm{U},{ }^{235} \mathrm{U},{ }^{238} \mathrm{U},{ }^{239} \mathrm{Pu} .{ }^{34}$ Also light $(Z<17)$ nuclei were probed via this transition. However, due to insufficient resolution at that time, only one parameter of the charge distribution (the r.m.s. radius) could be derived for higher transitions, 

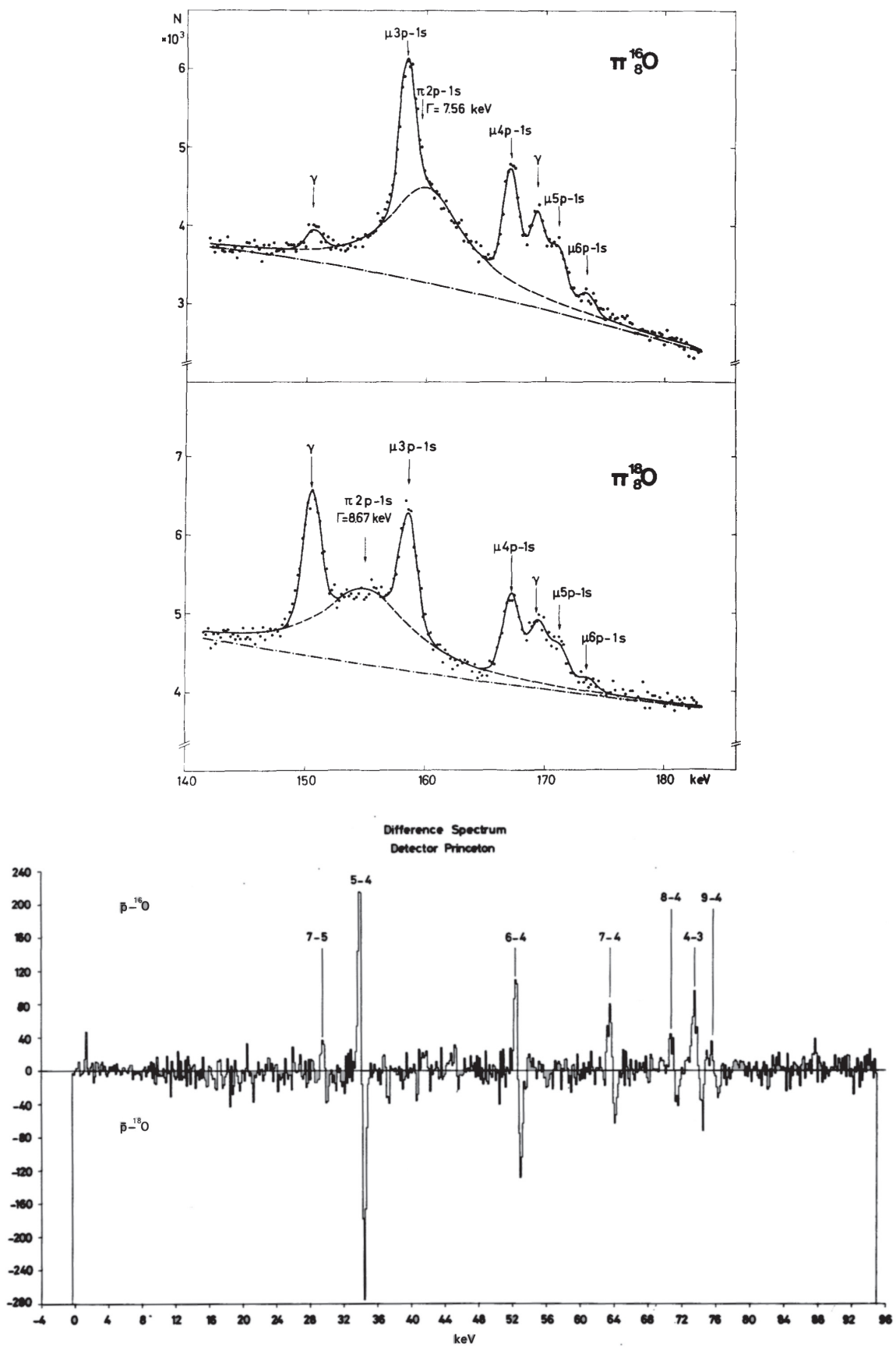

Fig. 2. Isotopic shifts in exotic atoms. Top: Pionic $2 \mathrm{p}-1 \mathrm{~s}$ transitions in ${ }^{16} \mathrm{O}$ and ${ }^{18} \mathrm{O} .{ }^{5}$ The broad pionic $2 \mathrm{p}-1 \mathrm{~s}$ transition and background muonic atomic transitions are visible (data published in 1967). Bottom: Antiprotonic oxygen: difference spectrum of $\overline{\mathrm{p}}-{ }^{16} \mathrm{O}$ and $\overline{\mathrm{p}}-{ }^{18} \mathrm{O}^{26}$ (data published in 1978). 
e.g. $3 d-2$ p. Since this parameter could be determined from the X-ray energy of the transition almost independently of the model of the charge distribution it was used as a proxy for the size of the charge distribution. The precision on the r.m.s. radii of the probed nuclei was surpassed by electron scattering experiments only for the lightest $(Z<8)$ nuclei.

Another way to probe the nuclear periphery relies on antiprotonic atoms. Neutron density distributions can be sampled in (heavy) nuclei by correlating measurements of their antiprotonic X-ray cascade with a radiochemical determination of the same nuclei after they have been exposed to antiproton capture and annihilation (and are consequently one mass unit lighter). The density distributions can be described via a two-parameter Fermi distribution which defines a halfdensity radius and a diffuseness parameter for both protons and neutrons. In a series of measurements, the PS209 experiment at LEAR investigated a range of 34 different nuclei, from ${ }^{40} \mathrm{Ca}$, to ${ }^{238} \mathrm{U}$ via both techniques. If proton distributions are constrained to the values from electron scattering or muonic-X-ray measurements, then the neutron density distribution is best reproduced in terms of a half-density radius compatible with that of the proton, but a significantly larger diffuseness ${ }^{36}$ in the case of neutron-rich nuclei.

While current research on exotic atoms at CERN is mainly focused on antiprotonic atoms, muonic exotic atoms with ever improved experimental precision continue to play an essential role elsewhere, sensitive perhaps even to physics beyond the standard model. ${ }^{37}$ One such area is that of muonic hydrogen. A $7 \sigma$ discrepancy separates the determination of the proton radius obtained through a measurement of the Lamb shift in muonic hydrogen, ${ }^{38,} 39$ at the CREMA experiment at PSI from that obtained from electron scattering and spectroscopic studies of atomic hydrogen and deuterium. This discrepancy — barring experimental errors — can only be accounted for by modifications at a very deep level (QED, lepton universality, change in the Rydberg constant), ${ }^{37}$ which illustrates the topicality of research on exotic atoms even today.

\section{Antiprotonic Atoms and Protonium}

The simplest antiprotonic atom is protonium, a bound state of an antiproton with a proton. These atoms are formed by slowing down antiprotons (by ionisation loss) in hydrogen. Once the antiproton is (almost) at rest, it will replace the electron of the hydrogen atom, in an orbit whose radius is that of the first Bohr radius of hydrogen, approximately $5.3 \times 10^{-9} \mathrm{~cm}$. The protonium thus formed will have a large angular momentum, $l$, and a principal quantum number, $n$, of about $\left(m_{p} / 2 m_{e}\right)^{1 / 2}$ ( $\sim 30)$, where $m_{p}$ and $m_{e}$ are the mass of the proton and of the electron. ${ }^{40}$ During de-excitation, $(n, l)$ quantum numbers are reshuffled: for formation of protonium in liquid hydrogen, Desai ${ }^{40}$ showed that annihilation of the proton-antiproton system would take place essentially exclusively from states with zero angular momentum 
(albeit with possibly high principal quantum number). This prediction depends on the likelihood of Stark mixing, however, and so is strongly density dependent: protonium formation and cascades in low density hydrogen (requiring of course low-momentum antiprotons) would be expected to contribute annihilations from $\mathrm{p}$ (or higher angular momentum) states.

Of course, antiprotonic atoms with heavier nuclei are also possible. The special case of antiprotonic helium will be discussed below, as measurement of transitions between its meta-stable states allow precision determinations of the antiproton's mass, charge and magnetic moment. In general however, antiprotonic atoms are short-lived, and are ideal probes of strong interaction effects. Atomic physics with antiprotons at CERN began with the first observation of antiprotonic atoms in $1970,{ }^{8}$ also providing the first spectroscopic measurement of the antiproton mass. Figure 3 shows the X-ray spectrum produced by stopping $14 \times 10^{6}$ antiprotons in a $\mathrm{Tl}$ target. Comparison between the measured and calculated transitions allowed the authors to give a $68 \%$ CL upper limit on any mass difference between protons and antiprotons of $\left(\left|m_{\mathrm{p}}-m_{\overline{\mathrm{p}}}\right|<0.5 \mathrm{MeV}\right)$, a relative precision of $5 \times 10^{-4}$. Only a consistency check on the equality of the magnetic moment of the proton and of the antiproton could be provided, the (limited) accuracy of the measurements precluding any quantitative statement at the time. In a subsequent experiment on antiprotonic X-rays of $\mathrm{P}, \mathrm{Cl}, \mathrm{K}$, of $\mathrm{Sn}, \mathrm{I}, \mathrm{Pr}$, and of $\mathrm{W}$, the same group ${ }^{41}$ explored more fully the effect of the strong interaction on the deepest lying bound states, and in addition to determining the reduction of some transitions, also observed the effect of the strong interaction via the line width and energy shift in one of the target nuclei, sulfur. These X-ray studies - as in the case of pionic atoms - also allowed probing isotopic effects, this time with antiprotons: the first measurements (still at

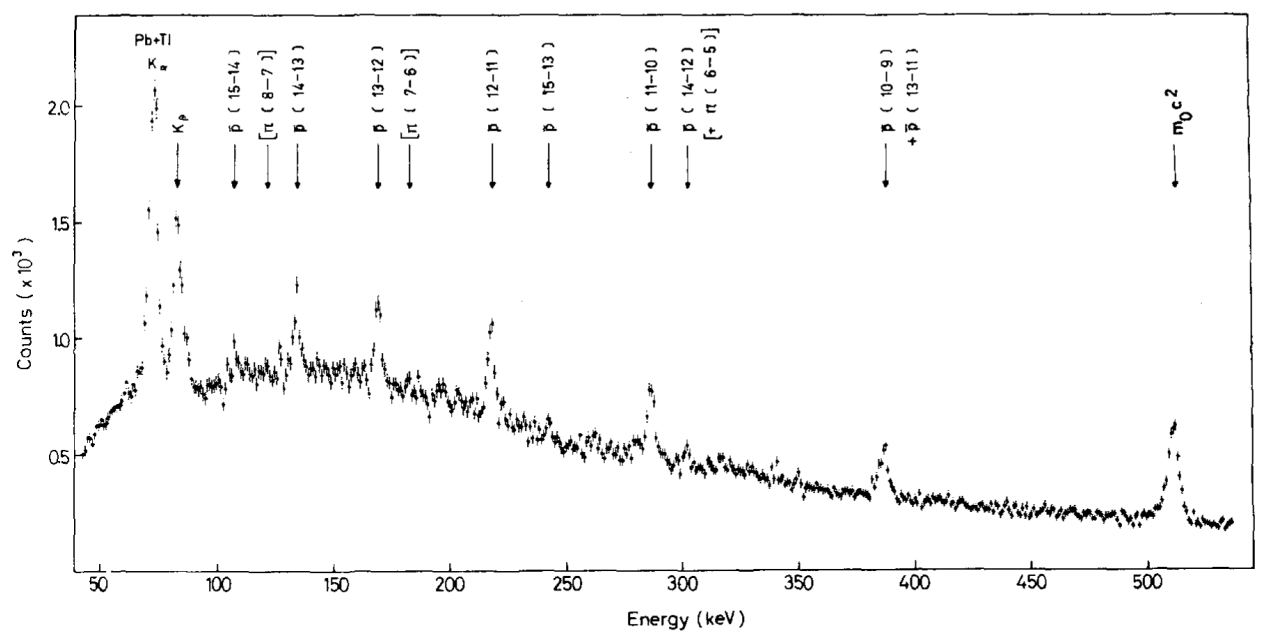

Fig. 3. X-ray spectrum of the first antiprotonic atom to be observed: $\bar{p}-{ }^{81} \mathrm{TI}$ obtained from $14 \times 10^{6}$ stopped antiprotons measured with a $10 \mathrm{~cm}^{3} \mathrm{Ge}(\mathrm{Li})$-detector. ${ }^{8}$ 
Table 1 Antiproton mass and magnetic moment measurements.

\begin{tabular}{lccccr}
\hline Quantity & Year & Value & Rel. precision & Method & Ref. \\
\hline$\left|m_{\mathrm{p}}-m_{\overline{\mathrm{p}}}\right|$ & 1970 & $<0.5 \mathrm{MeV}$ & $5 \times 10^{-4}$ & $\overline{\mathrm{p}}-\mathrm{Tl}$ & 8 \\
$\left|m_{\mathrm{p}}-m_{\overline{\mathrm{p}}}\right|$ & 1977 & $<0.05 \mathrm{MeV}$ & $5 \times 10^{-5}$ & $\overline{\mathrm{p}}-\mathrm{Zr}$ and $\overline{\mathrm{p}}-\mathrm{Y}$ & 25 \\
$m_{\overline{\mathrm{p}}} / m_{\mathrm{p}}$ & 1990 & $0.999999977(42)$ & $4 \times 10^{-8}$ & trapped $\overline{\mathrm{p}}$ & 74 \\
$m_{\overline{\mathrm{p}}} / m_{\mathrm{p}}$ & 1995 & $0.9999999995(11)$ & $1 \times 10^{-9}$ & trapped $\overline{\mathrm{p}}$ & 66 \\
$(q / m)_{\overline{\mathrm{p}}} /(q / m)_{\mathrm{p}}$ & 1999 & $-0.99999999991(9)$ & $5 \times 10^{-11}$ & trapped $\overline{\mathrm{p}}$ & 63 \\
\hline$\left(\mu_{\mathrm{p}}-\left|\mu_{\overline{\mathrm{p}}}\right|\right) / \mu_{\mathrm{p}}$ & 1972 & $(-0.04 \pm 0.1)$ & $3 \times 10^{-2}$ & $\overline{\mathrm{p}}-\mathrm{Pb}$ & 79 \\
$\left(\mu_{\mathrm{p}}-\left|\mu_{\overline{\mathrm{p}}}\right|\right) / \mu_{\mathrm{p}}$ & 2009 & $(2.4 \pm 2.9) \times 10^{-3}$ & $10^{-3}$ & $\overline{\mathrm{p}}-\mathrm{He}$ & 70 \\
$\mu_{\overline{\mathrm{p}}} / \mu_{\mathrm{p}}$ & 2013 & $-1.000000(5)$ & $5 \times 10^{-6}$ & trapped $\overline{\mathrm{p}}$ & 78 \\
\hline & & & &
\end{tabular}

the PS) of these isotope effects with antiprotonic atoms (Fig. 2) in $\overline{\mathrm{p}}-{ }^{16} \mathrm{O} /{ }^{18} \mathrm{O}$ provided important new information on the $\overline{\mathrm{p}}-\mathrm{n}$ interaction, ${ }^{26}$ allowing to separate the $\overline{\mathrm{p}}-\mathrm{n}$ and $\overline{\mathrm{p}}-\mathrm{p}$ scattering lengths, although some assumptions about the neutron distributions in the nuclear tail needed to be made.

It subsequently took several more years for more precise measurements of the transitions in antiprotonic atoms $(\overline{\mathrm{p}} \mathrm{Pb}$ and $\overline{\mathrm{p}} \mathrm{U})$ to result in improved measurements of antiproton parameters: the anomalous magnetic moment in $1972,{ }^{79}$ subsequently improved in $1975,{ }^{9}$ a measurement that also yielded a 10 -fold improvement over the first spectroscopic antiproton mass measurement at CERN. Further measurements, still at Brookhaven, ${ }^{25}$ of the fine structure splitting continued to improve the knowledge of the antiproton mass $\left(m_{\overline{\mathrm{p}}}=938.229 \pm 0.049 \mathrm{MeV}\right.$, Table 1$)$ but were still hampered by theoretical uncertainties and - more importantly — detector resolution.

The sensitivity of early measurements of antiprotonic He at the PS ${ }^{44}$ was not sufficient to detect the small fraction of long-lived $(\sim \mu \mathrm{s})$ meta-stable states discovered in 1991. ${ }^{43}$ These states opened the door to high precision laser-spectroscopic studies of the energy levels of the antiprotonic atoms by the PS205 (at LEAR, until $1996^{45}$ ) and the ASACUSA (at the AD) experiments. This methodological advance over resolution-limited X-ray detectors consequently improved the knowledge of the mass, charge (Section 4.1) and magnetic moment (Section 4.2) of the antiproton by many orders of magnitude beyond what measurements of the de-excitation cascade could provide.

\subsection{Protonium}

This simplest antiprotonic atom provides a particularly pure measurement of the $\bar{p}-p$ interaction via the usual energy level broadening and shift studies. Furthermore, it offers the very attractive possibility of tagging the initial state of $\overline{\mathrm{p}}-\mathrm{p}$ annihilation via detection of the corresponding X-ray transition. Knowing the quantum numbers of the initial state consequently had a significant impact on meson spectroscopy in the 1980s at CERN, by limiting the possible contributing 
waves to the Dalitz plot fits that were the workhorse of many experiments at LEAR. ${ }^{46}$ This in turn made it possible to better identify the quantum numbers of short-lived resonances produced in $\bar{p}-\mathrm{p}$ annihilations. It was only in 1978 that the first observation of (Balmer Series) X-rays from antiprotonic hydrogen took place $^{47}$ at the low-momentum antiproton line of the PS. Subsequent experiments on protonium benefitted greatly from CERN's dedicated antiproton program from 1980 onwards (Section 4), in particular from the background-free low energy antiproton beam provided by the LEAR facility.

A series of spectroscopic measurements of transitions between low-lying protonium bound states using different techniques (with different sensitivities and resolutions) took place in the first and second generation of LEAR experiments. PS171 (ASTERIX collaboration) identified the Lyman- $\alpha$ line, ${ }^{48}$ measured the strong interaction shift and broadening of the ground state of the $\bar{p}-\mathrm{p}$ atoms, ${ }^{49}$ and obtained the cascade time of antiprotons in gaseous hydrogen using a drift chamber as X-ray detector, ${ }^{16}$ a measurement extended by PS201 (Obelix collaboration) to a large range of $\mathrm{H}_{2}$ densities. ${ }^{17}$ Improved resolution was provided by a dedicated experiment (PS174) using a $\mathrm{Si}(\mathrm{Li})$ detector, ${ }^{50}$ which subsequent experiments using a much higher resolution spectrometers (PS175, using a cyclotron trap for formation of protonium at very low pressures ${ }^{51}$ or PS207, using CCD's coupled to a crystal spectrometer ${ }^{52}$ ) improved by several orders of magnitude. The results on the spin triplet and spin singlet strong interaction shifts and widths were instrumental in finessing the $\overline{\mathrm{N}}$ potential models and even uncovered problems (resolved since) with QED calculations for both hydrogen and other isotopes.

\section{Antiprotons}

Experiments on and with antiprotons at CERN started almost immediately after the required energy for producing them was available, and have carried on uninterruptedly since then, spawning new experimental facilities and opening new experimental areas on the way. The first facility capable of producing antiprotons at CERN was the Proton Synchrotron; completed in 1959, the first antiprotons were produced shortly afterwards. ${ }^{1}$ Experiments using the newly accessible antiprotonproton annihilations to investigate the time-like structure of the proton ${ }^{53}$ or in tandem with developments of large bubble chambers - as a testbed for measurements of cross sections and meson spectroscopy (from 1965) quickly started up at $\mathrm{CERN}^{54}$ and at Brookhaven's AGS. ${ }^{55}$ However, precision experiments on antiprotons and exotic atoms incorporating them could start in earnest only once a pure beam of antiprotons was available, requiring a dedicated facility for their production, storage and extraction.

This became possible through the invention of stochastic cooling (proposed in 1968 by S. van der Meer, published in 1972), ${ }^{56}$ and its successful test in the Initial Cooling Experiment (ICE) in $1978^{57}$ which demonstrated an increased beam 
density, as well as an extension of the beam lifetime from hours (without cooling to counteract Coulomb scattering on residual gas) to days. This test also allowed a dramatic improvement in a test of the CPT symmetry: the equality of lifetimes of protons and antiprotons. Prior to this test, the experimental lower limit on the lifetime of antiprotons (derived from bubble chamber tracks) was $120 \mu \mathrm{s}$; by inserting a simple antiproton production target upstream of the ICE set-up and cooling and storing a minute number of antiprotons in ICE, a 9-order of magnitude improved lower limit on the lifetime of antiprotons could be obtained. ${ }^{58}$

This fundamental advance allowed CERN's antiproton complex (Figs. 4 and 5) consisting of the antiproton production target, the Antiproton Accumulator (AA) and the Antiproton Collector (AC) and the low energy antiproton ring (LEAR) and relying on the Proton Synchrotron which produces $26 \mathrm{GeV} / c$ protons, to be proposed $^{59}$ and rapidly built (AA start-up in 1980, LEAR began operation in 1982, AC from 1987 onwards).

The development of antiproton trapping and electron cooling techniques by Gabrielse et al. ${ }^{60}$ in 1986 at CERN's dedicated antiproton experimental facility LEAR finally allowed carrying out precision experiments on trapped and cooled antiprotons and working towards the study of antihydrogen atoms first at LEAR

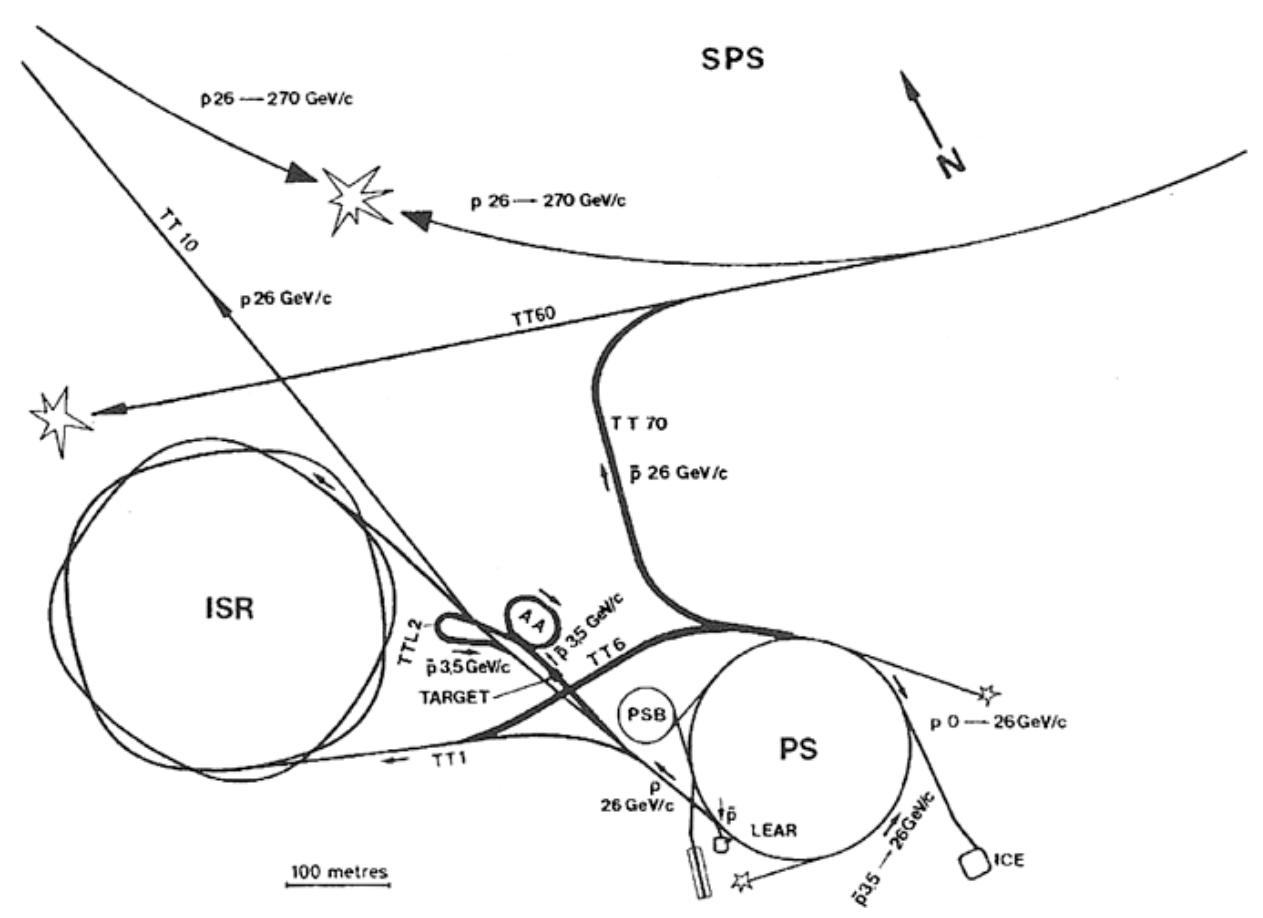

Fig. 4. Layout of the CERN accelerators in 1981. LEAR, still under construction in 1981, is also shown. ${ }^{58}$ From 2000 onwards, the AA was transformed into the Antiproton Decelerator, and now houses at CERN all experiments worldwide relying on low energy antiprotons. 

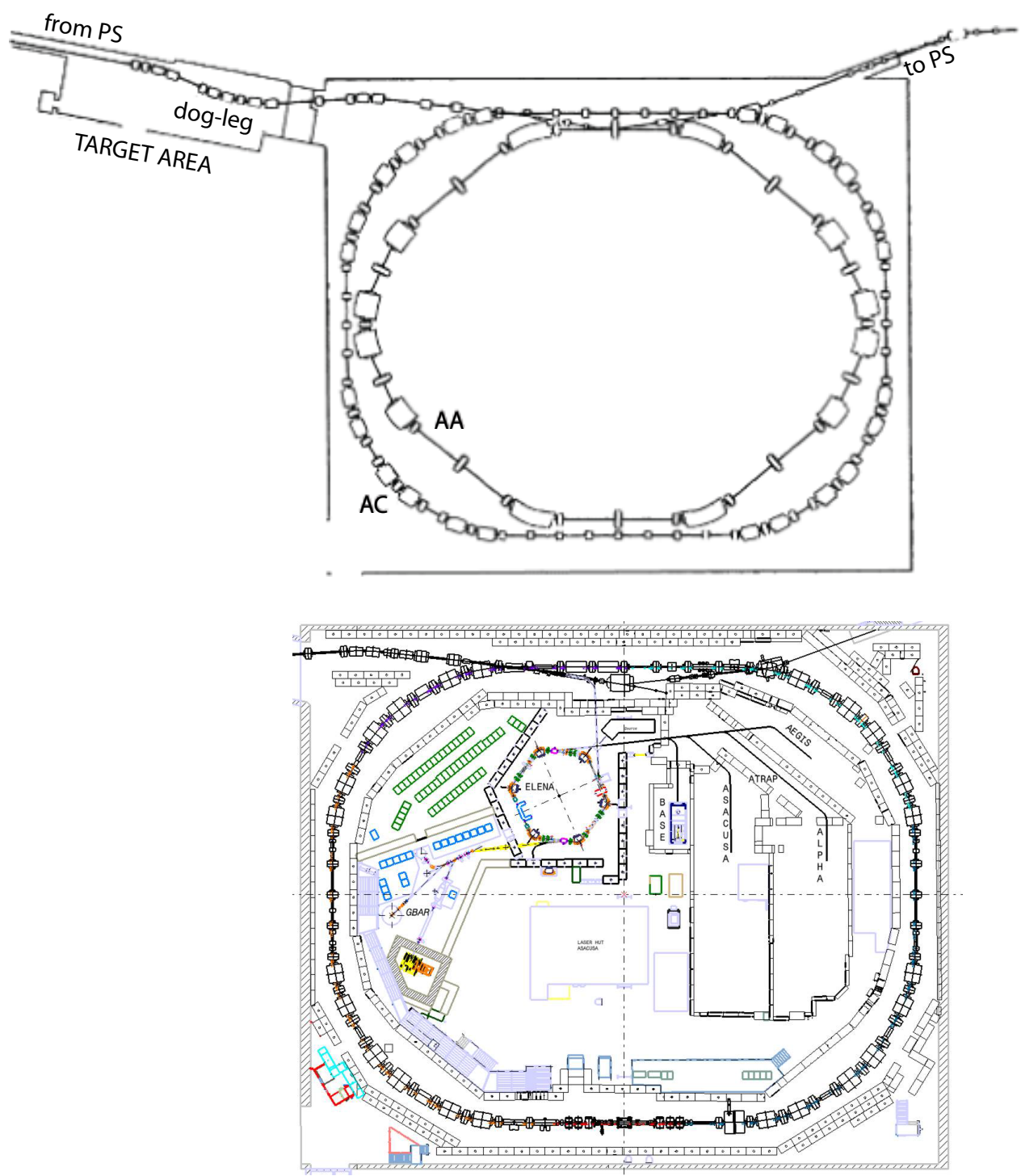

Fig. 5. Top: In 1986/87, the AC was built around the AA and a dog-leg incorporated into the injection line to diminish the flux of electrons and $\pi^{-}$reaching the hall. ${ }^{61}$ Bottom: AD layout with experimental areas (status at the end of 2014) with the future "Extra Low Energy Antiproton ring" (ELENA) indicated.

from 1986 to 1996, and - since 2000 - at the unique Antiproton Decelerator (AD) facility (transformed from the AC), which hosts all existing experiments requiring trapped antiprotons. Confinement of antiprotons in ion traps for seconds (or days) opened up major improvements in the determination of the antiproton's mass, charge, but also of its magnetic moment (although it took until 2012 to surpass the 
precision achievable in antiprotonic helium transitions). Furthermore, the formation and trapping of antihydrogen atoms, precision spectroscopy of antihydrogen, or precise measurements of the protonium energy levels without collisional broadening could also be envisaged, prepared and — by 2014 — partly achieved.

\subsection{Charge and mass: TRAP, ATRAP and ASACUSA}

A comparative measurement of the charge and mass of antiprotons and protons allows a direct test of CPT invariance. While exotic antiprotonic atoms had allowed reaching a relative precision of $10^{-5}$, further improvements were limited by the precision with which the energies of the transition could be measured as well as calculated. With the techniques (Fig. 6) for slowing, trapping, cooling and stacking of antiprotons now established by his PS196 collaboration, ${ }^{60,} 64$ Gabrielse's group rapidly carried out a first determination of the $q / m$ ratio of antiprotons ${ }^{74}$ with 100 simultaneously trapped antiprotons. For this, he measured their cyclotron frequencies in the Penning trap in which the antiprotons were confined and with this method achieved a factor of 1000 improvement in precision relative to the previous best values coming from exotic antiprotonic atoms. ${ }^{25,65}$ In rapid succession, they further refined and improved their sensitivity, reaching first a precision of $1 \mathrm{ppb}^{66}$ with a single trapped antiproton before extending the technique to a $90 \mathrm{ppt}$ precision (Table 1). The main systematics limiting the previous measurements were addressed in this final measurement at the LEAR complex: the use of an $\mathrm{H}^{-}$ion instead of a proton, and simultaneous confinement of the antiproton and the $\mathrm{H}^{-}$ion in the same trap (Fig. 6). This avoids non-reproducible electric trapping potentials, which had led to differences in the magnetic field experienced by the antiproton and proton of up to $1 \mathrm{ppb}$. As well, due to the ease with which $\overline{\mathrm{p}}$ and $\mathrm{H}^{-}$could be switched and probed, magnetic drifts affected the (alternating) measurements far less than earlier measurements.

At the same time, the PS205 experiment focused on precision measurements of transitions in antiprotonic helium $\left(\overline{\mathrm{p}} \mathrm{He}^{+}\right)$, benefiting from the co-temporaneous discovery $^{43}$ that these could form meta-stable states $(\tau \sim 3-4 \mu s)$, thus opening the window to inducing (via laser pulses) transitions between these meta-stable states and unstable states which would rapidly decay and produce a clear annihilation signal. The technique developed by PS205 ${ }^{67}$ is based on injecting a large number of antiprotons into gaseous helium. While short-lived states will annihilate within few ns, some long-lived meta-stable states may be populated and can be probed via a delayed laser pulse which - if it corresponds to a transition energy from a populated meta-stable state into a short-lived state - will lead to a short increase in the annihilation rate (Fig. 7). The significant advance over direct measurements of the emitted X-rays is that the precision is now limited mainly by the external laser system applied to stimulate the transitions. In a series of improvements of their apparatus and technique (frequency combs, doppler-free transitions, low density 

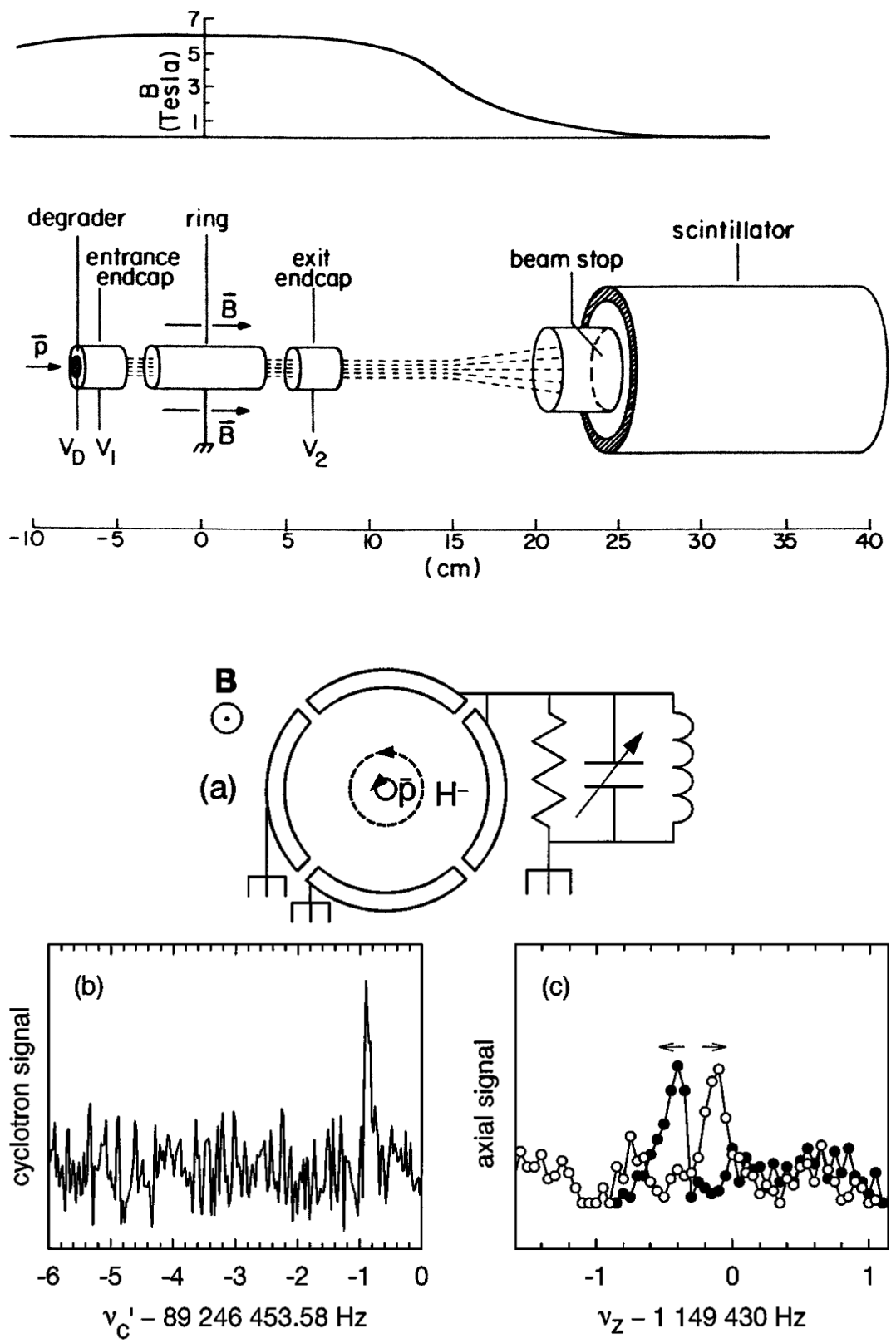

Fig. 6. Top: Outline of the $\overline{\mathrm{p}}$ trap electrodes and the scintillator. The direction of the homogeneous magnetic field is indicated by the arrow, its magnitude along the center axis is plotted above and important field lines are indicated by dashed lines. ${ }^{60}$ Bottom: (a) Central trap electrode, viewed along B, and the LCR detection circuit used to observe the signal (b) induced by free cyclotron motion. The driven axial signal (c) induced across a similar circuit, as the drive is stepped up or down in frequency every $4 \mathrm{~s}$, is delayed by a detector time constant. Thus $\nu_{z}$ (needed to $\pm 0.7 \mathrm{~Hz}$ to determine it to $600 \mathrm{ppb}$ in order to determine the cyclotron frequency $\nu_{c}$ to $90 \mathrm{ppt}$ ) is midway between the peaks. ${ }^{63}$ 


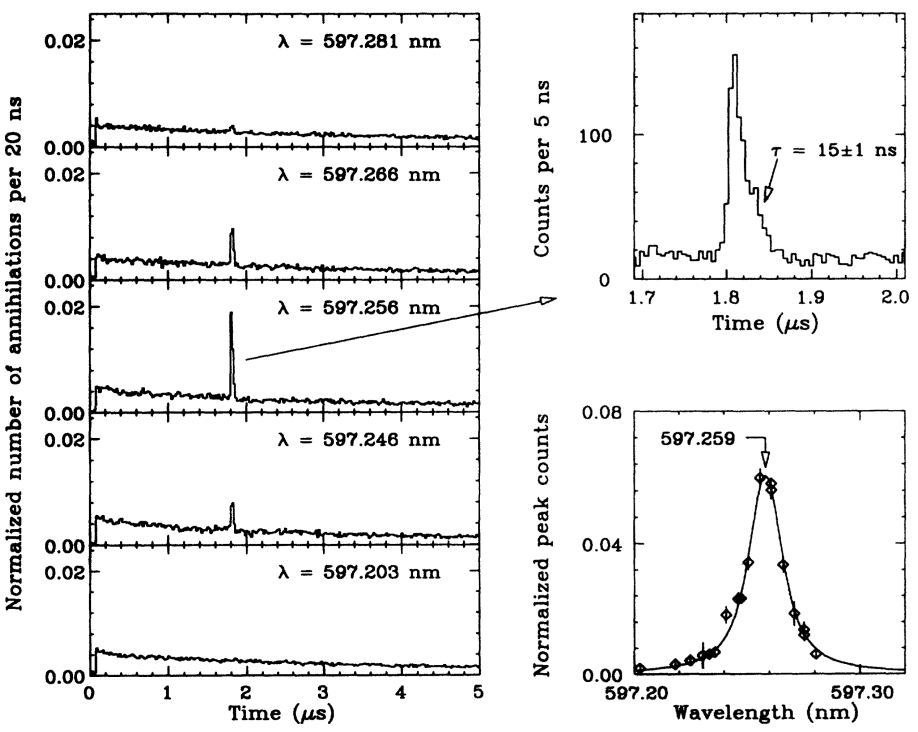

Auger dominated daughter state

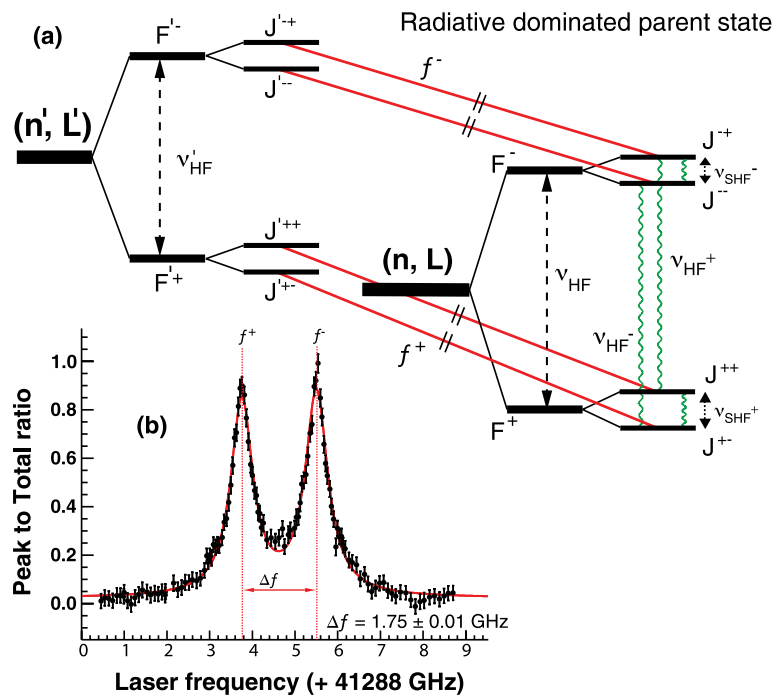

Fig. 7. Top: Observed time spectra of delayed annihilation of antiprotons with laser irradiation of various vacuum wavelengths near $597.2 \mathrm{~nm}$, normalised to the total delayed component. ${ }^{12}$ Spikes due to forced annihilation through the resonance transitions are seen at 1.8 ps. Upper right: Enlarged time profile of the resonance spike. A damping shape with a time constant of $15 \pm 1 \mathrm{~ns}$ is observed. Middle right: Normalised peak count versus vacuum wavelength in the resonance region, showing a central wavelength 597.259 \pm 0.002 and a FWHM $0.018 \mathrm{~nm}$. Bottom: (a) Schematic view of the level splitting of $\overline{\mathrm{p}} \mathrm{He}^{+}$for the $(n, l) \rightarrow(n-1, l+1)$ electric dipole transitions. ${ }^{70} \mathrm{The}^{\mathrm{T}}$ laser transitions $f^{+}$and $f^{-}$, from the parent to daughter states, are indicated by straight lines and the microwave transitions, between the quadruplets of the parent, by wavy ones. For this experiment $(n, L)=(37,35)$ and $\left(n^{\prime}, L^{\prime}\right)=(38,34)$. (b) Laser resonance profile demonstrating the two sharp peaks and HF laser splitting $\Delta f=f^{-}-f^{+}$. Although there are four SHF laser transitions only the HF transitions were resolved in this experiment (see Section 4.2). 
targets), the PS205 experiment (and the successor experiment AD-3/ASACUSA) have improved the knowledge of the transitions by several orders of magnitude, now reaching ppb precisions. In parallel, the crucial theoretical treatment of the $\bar{p} H e$ system has kept pace. ${ }^{68,69}$

This antiprotonic atomic system also allows a precision comparison of the charges of a particle and its antiparticle: different functional dependencies of the observables (i.e. the Rydberg constant of the exotic atom, and the cyclotron frequency of trapped negative particles) allow factorising charge and mass. A test to $10 \mathrm{ppm}$ of the equality of the charges and masses of antiprotons and protons ${ }^{24}$ combines measurements of transitions in antiprotonic atoms ${ }^{25}$ and of the cyclotron frequencies of trapped (anti)protons, ${ }^{74}$ and is limited only by the precision achievable in the exotic atoms' transitions.

With recent calculations of the theoretical transition frequencies $\left(O\left(\alpha^{7}\right)\right)$ at the level of $0.1 \mathrm{ppb}$ accuracy in $\mathrm{H}_{2}^{+}, \mathrm{HD}^{+}$and antiprotonic helium, ${ }^{71}, 72$ the bar has been raised once more, and further even more precise measurements planned by the ATRAP and ASACUSA collaborations should thus allow improving the knowledge of $m_{\bar{p}}$ and of $q_{\bar{p}}$ by another order of magnitude (or more).

\subsection{Magnetic moment: ATRAP, ASACUSA}

A first observation of (hyperfine) level splitting in antiprotonic helium (caused by the coupling of the orbital angular momentum of the antiproton to the spin of the remaining electron) was reported in 1997 by PS205. ${ }^{73}$ This splitting is of the order of $10 \sim 15 \mathrm{GHz}$. The interaction between the antiproton spin and that of the electron causes a further super-hyperfine and much smaller splitting of $150 \sim 300 \mathrm{MHz} .^{75}$ In order to see this minute splitting induced by the antiproton spin a far more complex microwave resonance experiment was required. A first detection of this hyperfine structure via a laser-microwave-laser resonance method was reported in $2002^{76}$ but only in $2009^{70}$ was the resolution of the transition sufficient (Fig. 7) to be able to extract the antiproton magnetic moment at the level of $10^{-3}$, establishing equality with the proton magnetic moment to the same precision.

While further improvements allowed detecting even the nuclear-spin induced splitting in antiprotonic ${ }^{3} \mathrm{He}^{+},{ }^{77}$ the precision that is achievable cannot rival with very recent measurements of the antiproton magnetic moment in traps. The first ever measurement of spin flips on a single trapped antiproton were carried out by the ATRAP collaboration ${ }^{78}$ in a specially-prepared Penning trap that adds a finely tuned magnetic bottle gradient to the trap's axial $B$ field. High resolution measurements of the resulting axial frequency shifts (stemming from the interactions of the cyclotron, magnetron and spin moments with $\Delta B$ ) allowed the ATRAP collaboration to improve the sensitivity on the comparison of the proton and antiproton magnetic moment by three orders of magnitude from the best exotic atom measurement to $4.4 \mathrm{ppm}$ (Table 1 ). 


\section{Antihydrogen}

Antihydrogen formation in traps was proposed already in $1986 .{ }^{60}$ Several production processes mixing antiprotons $(\overline{\mathrm{p}})$ with positrons $\left(\mathrm{e}^{+}\right)$or positronium $(\mathrm{Ps})$ are possible:

$$
\begin{array}{r}
\overline{\mathrm{p}}+\mathrm{e}^{+}+\mathrm{e}^{+} \rightarrow \overline{\mathrm{H}}+\mathrm{e}^{+}, \\
\overline{\mathrm{p}}+\mathrm{Ps} \rightarrow \overline{\mathrm{H}}+\mathrm{e}^{-}, \\
\overline{\mathrm{p}}+\mathrm{e}^{+} \rightarrow \overline{\mathrm{H}}+\gamma
\end{array}
$$

Unfortunately, these processes require high positron densities (three-body formation), production and transport of positronium towards trapped antiprotons, or have a very low cross-section (radiative formation). In 1994, before the positron accumulation technique based on radio-isotope decays developed in 1989 by the group of C. Surko ${ }^{80}$ had become advanced enough that the required numbers of positrons were routinely available for antihydrogen production in Penning traps to be attempted, an alternative route was proposed by members of the PS202 experiment ${ }^{81}$ to produce antihydrogen atoms in flight, using the interaction between the antiprotons stored in the LEAR ring and a jet-gas target consisting of Xe atoms. The production process of antihydrogen is then:

$$
\overline{\mathrm{p}} \mathrm{Z} \rightarrow \overline{\mathrm{p}} \gamma \gamma \mathrm{Z} \rightarrow \overline{\mathrm{p}} \mathrm{e}^{+} \mathrm{e}^{-} \mathrm{Z} \rightarrow \overline{\mathrm{H}} \mathrm{e}^{-\mathrm{Z}}
$$

where the requisite positrons are formed via the space-like interaction between photons formed by the antiprotons in the Coulomb field of the nucleus with charge $Z$. The experiment (called PS210) was carried out during 15 hours in the course of 1995. With an integrated luminosity (based on the number of antiprotons and the gas-target thickness) of $\mathcal{L}=5 \times 10^{33} \mathrm{~cm}^{-2}$, a total of 11 antihydrogen atom candidate events ${ }^{82}$ were detected (with an estimated background of 2 events). Although the high momentum of the antiproton beam $(1.94 \mathrm{GeV} / c)$ meant that the resulting antihydrogen atoms could not be studied, this proof-of-principle experiment gave great support to the subsequent modification of the AC into a full-fledged antiproton deceleration facility at which antihydrogen production in Penning traps, trapping of the produced antihydrogen atoms, and spectroscopy of these atoms could be attempted.

\subsection{Low energy antihydrogen: ATHENA, ATRAP}

Several proposals to carry out experiments at this new antiproton facility, the Antiproton Decelerator, planned for 1999, were submitted as soon as its construction had been decided. Two of them (P302: ATHENA and P306: ATRAP) specifically focused on the production and study of cold antihydrogen atoms, and were approved in 1997 with the names of AD1 and AD2. 
Both ATHENA and ATRAP were based on a similar experimental design: a multi-well Penning trap that would hold antiprotons and positrons simultaneously, and allow bringing them into contact in a controlled manner. In both experiments, the produced antihydrogen atoms - being neutral — would leave the formation region. Detection in the ATHENA case was via reconstruction of the annihilation vertex of the antiproton (through a two-layer double-sided silicon micro-strip detector that detected the annihilation pion trajectories) and of the opening angle (from the vertex) of the two $511 \mathrm{keV}$ photons produced in the positron annihilation: an excess at an opening angle of $\pi$ is the signal for antihydrogen annihilation. In the case of the ATRAP experiment, detection of $\overline{\mathrm{H}}$ was performed by field-ionising the produced atoms, and subsequently detecting the resulting, trapped, antiprotons. This scheme is well matched to the 3-body production process (expected to be dominant) of Eq. (4) which produces mostly highly excited states of antihydrogen.

The first observation of the production of antihydrogen via mixing of antiprotons and positrons was provided by ATHENA,${ }^{83}$ followed only a few weeks later by a confirmation of the process by ATRAP ${ }^{84}$ (Fig. 8). This second paper however went further, by giving a first indication of the production process, since field-ionisation of antihydrogen would not have allowed detecting deeply bound antihydrogen atoms, such as would have been produced in the competing radiative production process of Eq. (6).

Further information on the distribution of populated antihydrogen states could also be immediately obtained via the same field-ionisation detection scheme by varying the ionising field strength, ${ }^{85}$ confirming that antihydrogen formed by mixing
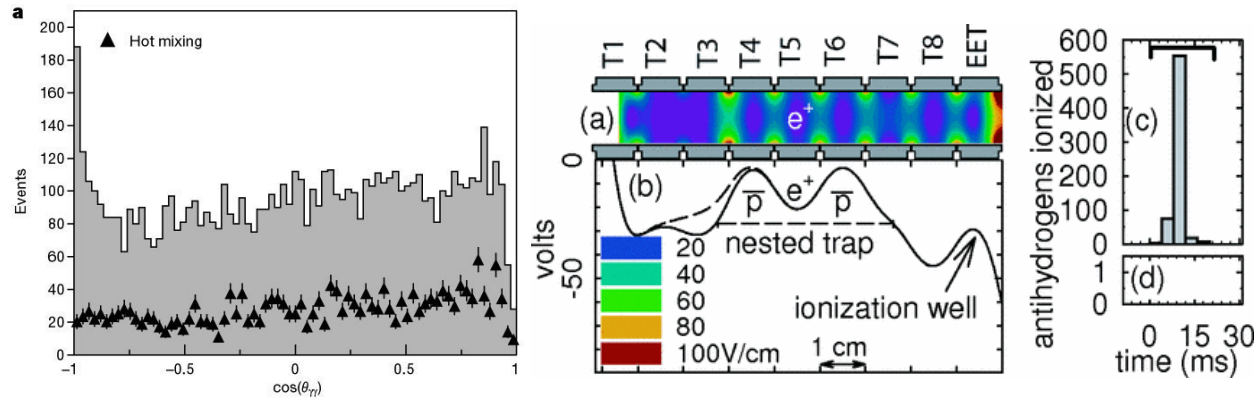

Fig. 8. Left: ATHENA experiment: Angular distribution between two detected photons from $\mathrm{e}^{+} \mathrm{e}^{-}$annihilation as determined from an antiproton annihilation vertex. The peak at $\cos (\theta)=-1$, but also the bulk of the distribution, corresponds to antihydrogen annihilations. The 'hot mixing' data correspond to mixing of cold antiprotons with RF-heated positrons, where no antihydrogen production can take place. ${ }^{83}$ Right: Electrodes for the ATRAP nested Penning trap, upon which a representation of the magnitude of the electric field that strips $\overline{\mathrm{H}}$ atoms is superimposed. (b) Potential on axis for positron cooling of antiprotons (solid line) during which $\overline{\mathrm{H}}$ formation takes place, with the (dashed line) modification used to launch $\overline{\mathrm{p}}$ into the well. (c) Antiprotons from $\overline{\mathrm{H}}$ ionisation are released from the ionisation well during a $20 \mathrm{~ms}$ time window. (d) No $\overline{\mathrm{p}}$ are counted when no $\mathrm{e}^{+}$are in the nested Penning trap. ${ }^{84}$ 
antiprotons and positrons in a nested-well Penning trap is mainly produced in Rydberg states. Subsequent analyses of plasma physics processes and simulations of the interaction of Rydberg antihydrogen atoms with the dense positron plasma confirmed and finessed this picture. ${ }^{86}$

With trapping of antihydrogen atoms the next goal after formation, measurements of the velocity distributions of the produced atoms unfortunately revealed that the antihydrogen atoms formed in the ATHENA and ATRAP experiments were far too energetic to be trapped. By employing a doublet of field-ionising electrodes and superimposing a temporal modulation on the first doublet, a velocitydependent transmission probability could be imposed on the continuous flux of antihydrogen atoms. The corresponding measurement by ATRAP ${ }^{87}$ confirmed the underlying problem that because the formation rate is determined by the relative velocity of antiprotons and positrons in the nested trap, even quite energetic antiprotons will have a velocity comparable to those of cold (but still fast, since light) positrons, consequently producing antihydrogen atoms with (relatively) high kinetic energy. The production of high temperature antihydrogen atoms in spite of the cryogenic environment in which they are formed was confirmed by a measurement by ATHENA of the axial distribution of antihydrogen annihilation vertices. ${ }^{88}$

One alternative to the nested well technique of producing antihydrogen, and that potentially could lead to much colder atoms being produced, as long as the antiprotons are far colder, is the charge exchange reaction of Eq. (5) whose crosssection scales with the Ps principal quantum number $n_{\mathrm{P}_{\mathrm{s}}}^{4}$. By producing and exciting positronium, it is thus possible to produce large amounts of Rydberg antihydrogen, with the additional benefit of having control of its Rydberg state. The large mass difference between the antiproton and the Ps entails that the kinetic energy of the formed antihydrogen is again dominated by that of the antiprotons. The scheme used by the ATRAP collaboration in $2004^{89}$ is explained in Fig. 9.

\subsection{Trapping: ALPHA, ATRAP}

Precision experiments on antihydrogen atoms benefit greatly from trapping them; if they are produced in nested electric potential wells (required to mix antiprotons with positrons), they will only survive for a few $\mu$ s after being formed before impacting on the walls of the electrodes forming the potential wells and annihilating. It is currently not possible to slow and cool energetic antihydrogen atoms formed randomly. Instead, trapping antihydrogen atoms relies on forming them inside a (neutral atom) trap, and at energies lower than the trap's potential. A magnetic minimum trap, which relies on the coupling of (anti)hydrogen atoms to magnetic fields via their small magnetic dipole moment can be formed by overlaying a transverse multipole (quadrupole, octupole) and two axial Helmholz-like coils. In its ground state, the magnetic moment of the antihydrogen atom is minimal (Rydberg states have a much larger dipole moment) but these are the states that need to be 

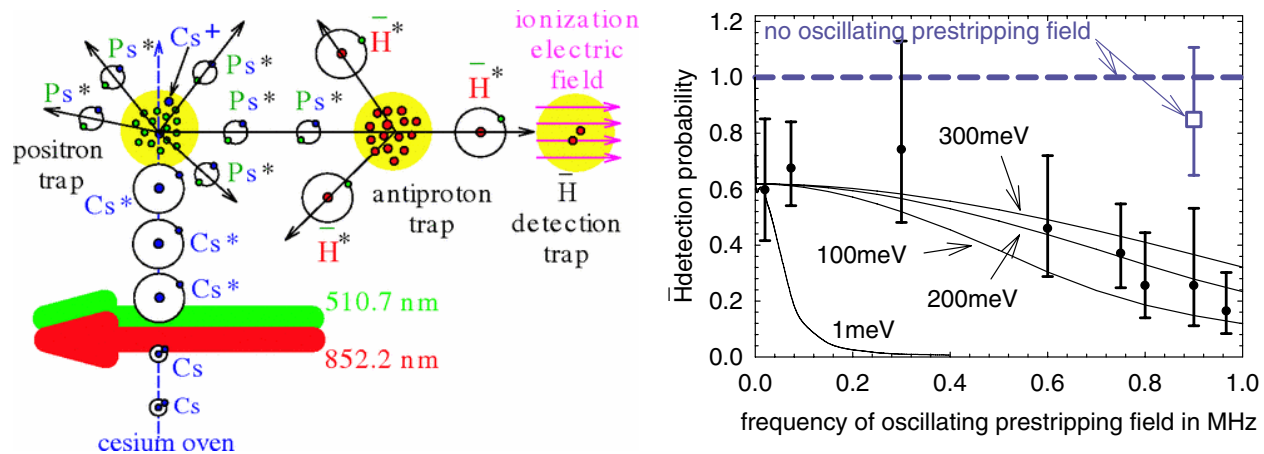

Fig. 9. Left: schematic for laser-controlled $\overline{\mathrm{H}}$ production: $\mathrm{Cs}$ atoms in a gas jet are excited into Rydberg states via two laser pulses (infrared: $852.2 \mathrm{~nm} ; 6 \mathrm{~S}_{1 / 2} \rightarrow 6 \mathrm{P}_{3 / 2}$; green: $510.7 \mathrm{~nm}$, $\left.6 \mathrm{P}_{3 / 2} \rightarrow 37 D\right)$; these Rydberg $\mathrm{Cs}$ atoms undergo a charge-exchange reaction with trapped cold positrons to form positronium (also in a Rydberg state); these neutral positronium atoms diffuse, and if they encounter antiprotons trapped nearby, will undergo a second charge exchange reaction to form Rydberg antihydrogen. ${ }^{89}$ Right: Transmission probability through a pre-fieldionising oscillating potential for high frequencies, only high velocity $\overline{\mathrm{H}}$ are transmitted: $300 \mathrm{meV}$ corresponds to $\approx 1000 \mathrm{~K}$.

trapped; the depth for these is $0.76 \mathrm{KT}^{-1}$. State-of-the-art systems achieve a trap depth of about 1 Tesla, and thus of $0.76 \mathrm{~K}$, corresponding to an antihydrogen kinetic energy of $65 \mu \mathrm{eV}$.

Even forming antihydrogen atoms in this challenging (magnetic) environment is a recent development. It is only in 2008 that the first antihydrogen atoms were formed in a quadrupolar ${ }^{90}$ or octupolar magnetic trap. Furthermore, antihydrogen atoms need to be formed at the lowest possible temperature, in order to trap even a fraction of the formed atoms. In 2010, the ALPHA collaboration reported on the first trapping of such ultra-cold antihydrogen atoms ${ }^{91}$ which correspond to a minute fraction of all atoms produced in their trap (Figs. 10(a)-10(c)); in a second paper, ${ }^{92}$ they furthermore showed that the trapped atoms had decayed into the ground state. Comparable results were also obtained by the ATRAP collaboration ${ }^{93}$ in 2012 , in spite of much slower trap release time constants (Fig. 10(d)).

\subsection{Spectroscopy: $A L P H A$}

The ultimate goal of antihydrogen experiments is to carry out precision spectroscopy. For this, two transitions are attractive: the transition between the ground state (1s) and the first excited states (2s) which can only decay via a two-photon transition, and has been measured in hydrogen ${ }^{94}$ to one part in $10^{14}$; and the hyperfine transition (HFS) in ground state antihydrogen, which - in hydrogen has been measured with a relative precision of $10^{-12}$, and can be measured - in antihydrogen — with a relative precision of $10^{-7}$ or better via microwave-cavity based methods pursued by the ASACUSA collaboration. 


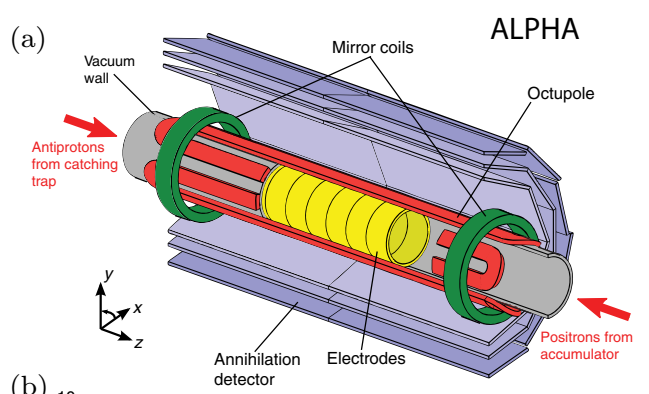

(b)

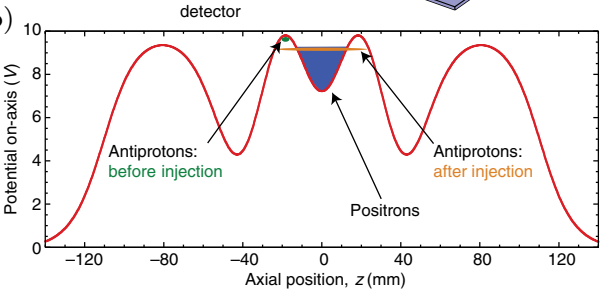

ALPHA

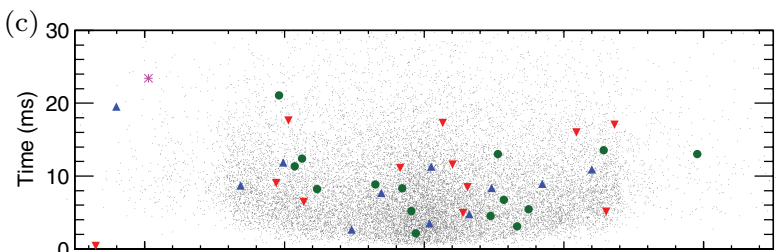

(d) 30

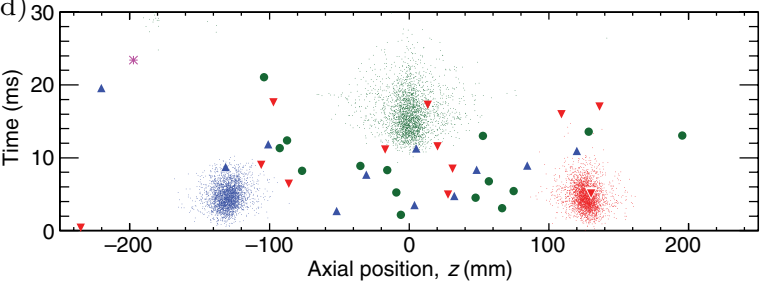

ATRAP
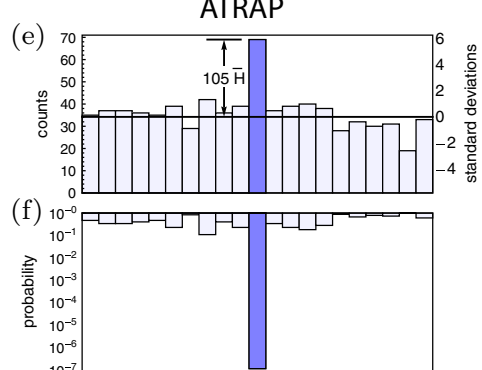

(g)

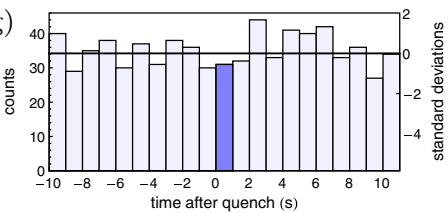

Fig. 10. Trapping of antihydrogen by ALPHA and ATRAP. Top: Antihydrogen synthesis and trapping region of the ALPHA apparatus (a) and nested-well potential used to mix antiprotons and positrons (b).

Bottom left: Measured $t-z$ distribution of annihilations obtained for three (red, green, blue) different experimental conditions (to differentiate trapped antihydrogen atoms from trapped antiprotons) during the opening of the ALPHA magnetic trap. Colored symbols are data, the grey dots are simulations for antihydrogen atoms (c); the same data are shown in figure (d), this time in comparison to expected distributions for antiprotons (tiny coloured dots) that could have been trapped instead of antihydrogen atoms, for three different experimental conditions. ${ }^{91}$ The colour codes are the same for data and simulations.

Bottom right: Detected antihydrogen annihilations after trap release at $t=0$ in the ATRAP experiment. The solid line at 35 counts corresponds to the average cosmic ray counting rate. (e) detected annihilation rate as a function of time; (f) probability that cosmic rays produce the observed counts or more; (g) control sample showing no signal during the trap quench. ${ }^{93}$ 
To date, a single spectroscopic measurement of antihydrogen has been carried out. The ALPHA collaboration focused first on the (HFS) microwave transition. ${ }^{95}$ Although the energy levels, and thus their splitting, of the trapped antihydrogen atoms depend on the position of the atom within the magnetic potential well, the field minimum of 1 Tesla ensures that no hyperfine splitting below that of the value at $1 \mathrm{~T}$ can take place. By exposing trapped antihydrogen atoms to a broad-band microwave radiation (15 MHZ around this minimum), the ALPHA collaboration was able to induce spin-flips in the trapped atoms, which then - because they were now in an un-trapped configuration - could be detected through annihilation. Figure 11 shows the Zeeman splitting, the spin-flip transition line shapes and microwave scan windows (two possible transitions), and the rate of detected antihydrogen atoms for 15-s scans over each window.
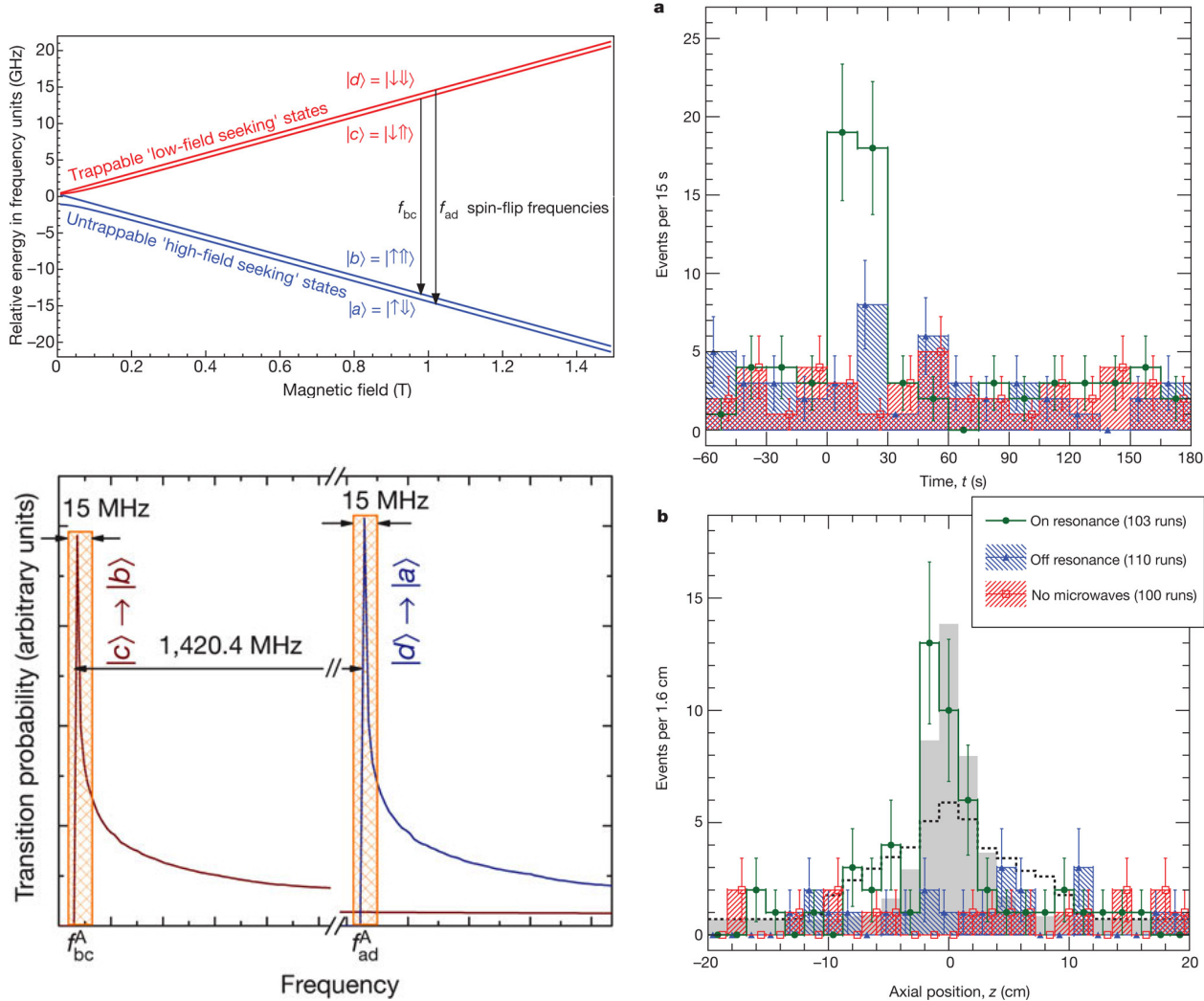

Fig. 11. Left: Breit-Rabi diagram of the hyperfine energy levels of ground state antihydrogen. below: Spin-flip transition line shapes and microwave scan windows for the $|c\rangle \rightarrow|b\rangle$ and the $|d\rangle \rightarrow|a\rangle$ transitions. Right: Number of detected antihydrogen annihilations before, during and after application of the microwaves (top) and axial position of the detected annihilations for $0<t<30 \mathrm{~s}$ (bottom) in the ALPHA experiment. ${ }^{95}$ 


\subsection{Gravity: AEgIS, GBAR}

The latest antihydrogen experiments at CERN aim at testing another fundamental symmetry: the weak equivalence principle. While experiments with the goal of measuring the behavior of matter and antimatter in the Earth's gravitational field have been contemplated before, the weakness of the gravitational interaction, and the impossibility of sufficiently shielding remnant electric and magnetic interactions for charged (anti)particles has hindered their realisation. Several groups have proposed experiments using neutral antihydrogen atoms as gravitational probes. The first such experiment (currently undergoing commissioning) is the AEgIS/AD-6 experiment. $^{96}$ It aims to produce a moderately focused pulsed horizontal beam of antihydrogen atoms, whose parabolic trajectory can be measured via a high resolution annihilation detector (a silicon-photographic emulsion hybrid). Two periodic gratings (a classical moiré deflectometer) ${ }^{97}$ are employed to produce a spatially modulated distribution of transmitted atoms. Since the vertical shift of the periodic pattern depends on the time during which a set of monoenergetic atoms fall, each atom's velocity is determined by forming all atoms simultaneously (through charge exchange between laser-excited positronium and ultra-cold antiprotons) and measuring the arrival time of the atom (in the annihilation detector) together with its vertical position.

A second method has been proposed by the GBAR collaboration: ${ }^{98}$ by interacting a low energy beam of antiprotons with a high-density cloud of positronium atoms, a dual charge exchange process (as described in Section 5.1), first forming ground state antihydrogen, and subsequently the bound $\overline{\mathrm{H}}^{+}$ion, a stable and positively charged antihydrogen ion is formed. Trapping and interacting this positive anti-ion with other laser cooled cations (e.g. $\mathrm{Cs}^{+}$) allows a first pre-cooling before a final cooling step with laser-cooled $\mathrm{Be}^{+}$to $\mu \mathrm{K}$ is carried out. Finally, the $\overline{\mathrm{H}}^{+}$is laser-ionised; the neutral $\overline{\mathrm{H}}$ 's free fall time from the trap to a detector measures its gravitational behavior.

Lastly, trapped antihydrogen atoms can also be released, and - if they are sufficiently cold - their subsequent free-fall behavior can be investigated. While first attempts $^{99}$ by the ALPHA experiment do not yet have the necessary sensitivity at several $100 \mathrm{mK}$, the temperatures of the (neutral, uncooled) trapped atoms are still far too high - this method may well become competitive, should laser cooling of antihydrogen atoms succeed in the coming years.

\section{References}

1. G. von Dardel et al., Phys. Rev. Lett. 5, 333 (1960).

2. A. Wightman, Phys. Rev. 77, 521 (1950).

3. M. Leon and H. Bethe, Phys. Rev. 127, 636 (1962).

4. P. Brix et al., Phys. Lett. 1, 56 (1962). 
5. G. Backenstoss et al., Phys. Lett. B 25, 365 (1967).

6. G. Backenstoss et al., Phys. Lett. B 32, 399 (1970).

7. G. Backenstoss et al., Phys. Lett. B 33, 230 (1970).

8. A. Bamberger et al., Phys. Lett. B 33, 233 (1970).

9. E. Hu et al., Nucl. Phys. A 254, 403 (1975).

10. E. Fermi and E. Teller, Phys. Rev. 72, 399 (1947).

11. L. I. Ponomarev, Ann. Rev. Nucl. Sci. 23, 395 (1975).

12. N. Morita et al., Phys. Rev. Lett. 72, 1180 (1994).

13. T. B. Day, G. A. Snow and J. Sucher, Phys. Rev. Lett. 3, 61 (1959).

14. R. Knop et al., Phys. Rev. Lett. 14, 767 (1965).

15. R. A. Burnstein et al., Phys. Rev. Lett. 15, 639 (1965).

16. G. Reifenröther et al., Phys. Lett. B 214, 325 (1988).

17. A. Bianconi et al., Phys. Lett. B 487, 224 (2000).

18. R. Schafer, Phys. Rev. 163, 1451 (1967).

19. R. Kunselman, Phys. Lett. B 34, 485 (1971).

20. S. C. Cheng et al., Nucl. Phys. A 254, 381 (1975).

21. G. Dugan et al., Nucl. Phys. A 254, 396 (1975).

22. G. Backenstoss et al., Phys. Lett. B 36, 403 (1971).

23. G. Backenstoss et al., Phys. Lett. B 43, 431 (1973).

24. R. Hughes and B. Deutsch, Phys. Rev. Lett. 69, 578 (1992).

25. P. Robertson et al., Phys. Rev. C 16, 1945 (1977).

26. H. Poth et al., Nucl. Phys. A 294, 435 (1978).

27. G. Backenstoss et al., Phys. Lett. B 38, 181 (1972).

28. W. Weise and L. Tauscher, Phys. Lett. B 64, 424 (1976).

29. B. Adeva et al., Phys. Lett. B 704, 24 (2011).

30. B. Adeva et al., Phys. Lett. B 674, 11 (2009).

31. J. Schweizer, Phys. Lett. B 587, 33 (2004).

32. H. Acker et al., Nucl. Phys. 87, 1 (1966).

33. G. Backenstoss et al., Nucl. Phys. 62, 449 (1965).

34. S. A. De Wit et al., Nucl. Phys. 87, 657 (1967).

35. G. Backenstoss et al., Phys. Lett. B 25, 547 (1967).

36. A. Trzcinska et al., Phys. Rev. Lett. 87, 082501 (2001).

37. R. Pohl et al., Annu. Rev. Nucl. Part. Sci. 63, 175204 (2013).

38. R. Pohl et al. (CREMA Collaboration), Nature 466, 213 (2010).

39. A. Antognini et al. (CREMA Collaboration), Science 339, 417 (2013).

40. B. Desai, Phys. Rev. 119, 1385 (1960).

41. G. Backenstoss et al., Phys. Lett. B 41, 552 (1972).

42. J. Fox et al., Phys. Rev. Lett. 29, 193 (1972).

43. M. Iwasaki et al., Phys. Rev. Lett. 67, 1246 (1991).

44. H. Poth et al., Phys. Lett. B 76, 523 (1978).

45. F. Hartmann, Hyperfine Int. 119, 175 (1999).

46. H. Koch, Hadron physics with antiprotons, in Proc. of the Int. School of Physics "Enrico Fermi", Course CLVIII, eds. T. Bressani, A. Filippi and U. Wiedner (IOS Press, 2005), p. 305 ff., DOI: 10.3254/1-58603-526-6-305.

47. E. G. Auld et al., Phys. Lett. B 77, 454 (1978).

48. S. Ahmad et al., Phys. Lett. B 157, 333 (1985). 
49. M. Ziegler et al., Phys. Lett. B 206, 151 (1988).

50. T. P. Gorringe et al., Phys. Lett. B 162, 71 (1985).

51. M. Augsburger et al., Nucl. Phys. A 658, 149 (1999).

52. D. Gotta et al., Nucl. Phys. A 660, 283 (1999).

53. M. Conversi et al., Phys. Lett. 5, 195 (1963).

54. U. Amaldi et al., Nuovo Cimento, 34, 825 (1964).

55. N. Barash et al., Phys. Rev. 139, B1659 (1965).

56. S. van der Meer, CERN Int. Report ISR- PO/72-31 (1972).

57. G. Carron et al., Phys. Lett. B 77, 353 (1978).

58. S. Gilardoni and D. Manglunki (eds.) Fifty Years of the CERN Proton Synchroton, Volume II, CERN-2013-005 (CERN, Geneva, 2013).

59. K. Killian, U. Gastaldi and D. Möhl, CERN/PS/DL 77-19.

60. G. Gabrielse et al., Phys. Rev. Lett. 57, 2504 (1986).

61. CERN report CERN/PS/86-30.

62. V. Chohan (ed.), CERN report CERN-2014-002.

63. G. Gabrielse et al., Phys. Rev. Lett. 82, 3198 (1999).

64. G. Gabrielse et al., Phys. Rev. Lett. 63, 1360 (1989).

65. B. Roberts, Phys. Rev. D 17, 358 (1978).

66. G. Gabrielse et al., Phys. Rev. Lett. 74, 3544 (1995).

67. H. Torii et al., Nucl. Inst. Meth. A 396, 257 (1997).

68. V. I. Korobov, Phys. Rev. A 54, 1749 (1996).

69. V. I. Korobov et al., Hyperfine Int. 194, 15 (2009).

70. T. Pask et al. (ASACUSA Collaboration), Phys. Lett. B 678, 55 (2009).

71. V. I. Korobov et al., Phys. Rev. Lett. 112, 103003 (2014).

72. V. I. Korobov et al., Phys. Rev. A 89, 032511 (2014).

73. E. Widmann et al., Phys. Lett. B 404, 15 (1997).

74. G. Gabrielse et al., Phys. Rev. Lett. 65, 1317 (1990).

75. D. Bakalov and V. I. Korobov, Phys. Rev. A 57, 1662 (1998).

76. E. Widmann et al., Phys. Rev. Lett. 89, 243402 (2002).

77. S. Friedreich et al. (ASACUSA Collaboration), J. Phys. B 46, 125003 (2013).

78. G. Gabrielse et al., Phys. Rev. Lett. 110, 130801 (2013).

79. J. D. Fox et al., Phys. Rev. Lett. 29, 193 (1972).

80. C. Surko et al., Phys. Rev. Lett. 62, 901 (1989).

81. G. Baur et al., ERN/SPSLC 94-29, P283 (1994).

82. G. Baur et al., Phys. Lett. B 368, 251 (1996).

83. M. Amoretti et al. (ATHENA Collaboration), Nature 419, 456-459 (2002).

84. G. Gabrielse et al., Phys. Rev. Lett. 89, 213401 (2002).

85. G. Gabrielse et al., Phys. Rev. Lett. 89, 233401 (2002).

86. S. Jonsell et al., J. Phys. B: At. Mol. Opt. Phys. 42, 215002 (2009).

87. G. Gabrielse et al., Phys. Rev. Lett. 93, 073401 (2004).

88. N. Madsen et al. (ATHENA Collaboration), Phys. Rev. Lett. 94, 033403 (2005).

89. C. H. Storry et al. (ATRAP Collaboration), Phys. Rev. Lett. 93, 263401 (2004).

90. G. Gabrielse et al. (ATRAP Collaboration), Phys. Rev. Lett. 100, 113001 (2008).

91. G. B. Andresen et al. (ALPHA Collaboration), Nature 468, 673676 (2010).

92. G. B. Andresen et al. (ALPHA Collaboration), Nature Physics, 7, 558 (2011).

93. G. Gabrielse et al., Phys. Rev. Lett. 108, 113002 (2012). 
94. M. Niering et al., Phys. Rev. Lett. 84, 54965499 (2000).

95. C. Amole et al., Nature 483, 439 (2012), doi:10.1038/nature10942.

96. CERN-SPSC-2007-017, http://cds.cern.ch/record/1037532/files/spsc-2007-017.pdf.

97. S. Aghion et al., Nature Commun. 5, 4538 (2014), doi: 10.1038/ncomms5538.

98. CERN-SPSC-2011-029, http://cds.cern.ch/record/1386684/files/SPSC-P-342.pdf.

99. The ALPHA Collaboration and A. E. Charman Nature Commun. 4, 1785 (2013), doi: $10.1038 /$ ncomms 2787 . 